\title{
Biomarker indicators of elastin degradation in asymptomatic alpha-1 antitrypsin deficiency
}

\author{
Tatsiana Beiko ${ }^{1 *}$, Shuren $\mathrm{Ma}^{2}$, Charlie Strange ${ }^{1}$ and Gerard M Turino ${ }^{2}$ \\ ${ }^{1}$ Division of Pulmonary and Critical Care Medicine, Medical University of South Carolina, Charleston, USA \\ ${ }^{2}$ James P. Mara Center for Lung Disease, Division of Pulmonary, Department of Medicine, Mount Sinai, New York, USA
}

\begin{abstract}
Background: Increased serum desmosine (DES) and isodesmosine (IDES) levels occur in individuals with alpha-1 antitrypsin deficiency (AATD). Small series have confirmed the association with emphysema in moderate COPD.

Methods: The QUANTitative lung CT UnMasking emphysema progression in AATD (QUANTUM-1) study was a multicenter, prospective 3-year study of 49 $\mathrm{PiZZ}$ individuals with normal lung function. All received serial chest computed tomography (CT), spirometry and serum collection. VIDA analysis software defined the baseline 15th percentile density (PD15). Least squares regression of FEV1 values defined FEV1 slope. We analyzed correlations between demographics, FEV1 decline $(\mathrm{n}=49)$, and PD15 slope $(\mathrm{n}=31)$.

Results: The 49 participants had six study visits during which DES/IDES was measured. Despite normal FEV1\% predicted, mild emphysema was present on visual analysis. Baseline serum DES/IDES did not correlate with baseline PD15 ( $\left.\mathrm{r}^{2}=0.02, p=0.32\right)$. Baseline and average DES/IDES were highly correlated with patient age $\left(\mathrm{r}^{2}=0.29\right.$ and $0.42, p<0.0001$ for both). The mean DES/IDES averaged per patient over 3 years was $0.30 \mathrm{ng} / \mathrm{ml}$, higher than historical controls from the normal population $(0.19 \mathrm{ng} / \mathrm{ml})$. The mean DES/IDES over 3 years trended in the same direction as PD15 slope $\left(\mathrm{r}^{2}=0.06, p=0.16\right)$.

Conclusions: Mean DES/IDES was higher than normal in PiZZ individuals with FEV1 $\geq 80 \%$. DES/IDES failed to correlate with baseline PD15, PD15 decline, or FEV1 slope. The consistency of the 6 DES/IDES measurements in each patient adds knowledge about test to test variability of DES/IDES despite study limitations that include small size and lack of concurrent PiMM controls. Despite the presence of near normal pulmonary function in alpha-1 antitrypsin deficient patient's elevations of DES/IDES indicate increased elastin degradation which can progress and warrants preventive therapies.
\end{abstract}

\begin{abstract}
Abbreviations: DES: Desmosine; IDES: Isodesmosine; AATD: Alpha-1 Antitrypsin Deficiency; COPD: Chronic Obstructive Pulmonary Disease; QUANTUM-1: QUANTitative lung CT UnMasking emphysema progression in AATD; PiZZ: Protease Inhibitor ZZ; CT: Computed Tomography; PD15: $15^{\text {th }}$ Percentile Density; FEV1: Forced Expiratory Volume in 1 second; AAT: Alpha-1 Antitrypsin; NE: Neutrophil Elastase; BMI: Body Mass Index; ECLIPSE: Evaluation of COPD Longitudinally to Identify Predictive Surrogate Endpoints; post-BD: post-bronchodilator; NHANES III: National Health and Nutrition Examination Survey III; VIDA: Volumetric Imaging Display and Analysis; TLC: Total Lung Volume; FVC: Forced Vital Capacity; GOLD: Global Initiative for Chronic Obstructive Lung Disease; DLCO: Diffusing Capacity of the Lung for Carbon Monoxide; HU: Hounsfield Units; SPIROMICS: SubPopulations and InteRmediate Outcome Measures In COPD Study; CVD: Cardiovascular Disease; RAPID: Randomized placebo-controlled trial of Augmentation therapy in alpha-1 Proteinase Inhibitor Deficiency study
\end{abstract}

\section{Introduction}

Alpha-1 antitrypsin deficiency (AATD) is a genetic disorder resulting in the development of pulmonary emphysema. While emphysema is the most common pulmonary manifestation in AATD, bronchitis, asthma, and bronchiectasis also occur. AATD presents at a younger age compared to usual COPD, and have enhanced susceptibility to effects of tobacco, dusts, and fumes [1].

The serum level of alpha-1 proteinase inhibitor, otherwise known as alpha-1 antitrypsin (AAT) is determined by each of the inherited alleles on SERPINA1, the gene for AAT. The function of AAT is to inhibit the proteolytic enzyme neutrophil elastase (NE), increased levels of which are observed with smoking, inflammation, infections, and inhaled fumes. NE is produced by neutrophils and can degrade elastin connective tissues in the human body. Unopposed NE activity occurs in AATD because of deficient inhibition offered by AAT.

Elastin is a major component of the lung alveolar and airway structure and its degradation alters lung matrix structure and lung mechanics. Desmosine (DES) and isodesmosine (IDES) are amino acids that are released during degradation of mature elastin fragments in humans. DES/IDES are two crosslinking amino acids which occur only in elastin and are released into the circulation when mature elastin is degraded.

Animal studies have correlated urinary DES levels and airspace enlargement in models of emphysema [2,3]. Subsequently, human studies using urine, and blood DES/IDES in COPD [4,5] have shown

${ }^{\star}$ Correspondence to: Tatsiana Beiko, Division of Pulmonary and Critical Care Medicine, Medical University of South Carolina, 96 Jonathan Lucas St CSB 816 MSC 630, Charleston, SC, 29407, USA, Tel: +1 (843) 792 6833; E-mail: beiko@ musc.edu

Key words: emphysema, desmosine, isodesmosine, alpha-1 antitrypsin, lung densitometry

Received: September 03, 2018; Accepted: September 21, 2018; Published: September 25, 2018 
correlations with disease progression. Prior research established that DES/IDES levels are elevated in individuals with COPD and smokers. Small series have confirmed the association with emphysema presence and progression in patients with moderate COPD, both AATDassociated [6] and AATD-replete. Subsequent studies confirmed stronger relationships between DES and several lung function variables in COPD individuals compared to those without COPD diagnosis after adjustments for age, sex, height, body mass index (BMI), and smoking were made [7]. Additionally, in a subgroup of individuals with stable COPD but reduced lung diffusing capacity, serum DES was elevated [8]. COPD exacerbations also raise urinary DES levels [8].

Long term intravenous augmentation therapy for severe AATD has been licensed since 1987 based on the evidence that it restores the protective levels of AAT in blood and lung epithelial fluid [9]. Studies evaluating the effects of augmentation therapy on the DES/IDES levels have reported various results [10-13]. One study using a new method of analysis of DES/IDES showed statistically significant reductions of DES/IDES in plasma and bronchoalveolar lavage fluid in patients receiving augmentation therapy [12]. The RAPID study has revealed that augmentation therapy delays the progression of radiographic emphysema in AATD [14]. Further confirmation has come from the evaluation of the RAPID serum DES/IDES levels as correlations of CT density decline and these biomarkers were clearly demonstrated [15].

Most of the available data supports the notion that DES/IDES levels are robust biomarkers in specific subgroups of individuals. These subgroups include those actively smoking, COPD with higher symptom burden, and AATD individuals. In Evaluation of COPD Longitudinally to Identify Predictive Surrogate Endpoints (ECLIPSE), differences in DES levels between healthy individuals and COPD individuals were present after adjustment for smoking, gender, age, and BMI [16]. Most agree that AATD-COPD has the highest levels of DES/IDES [17] even when adjusted for the degree of smoking and spirometric impairment.

However, little is known about the DES/IDES levels when lungs are near normal in early AATD. Additionally, longitudinal variability in DES/IDES has been incompletely studied. We analyzed a large serum AATD biorepository "QUANTitative computed tomography UnMasking emphysema progression in alpha-1 antitrypsin deficiency" (QUANTUM-1) study. This multicenter 3-year prospective study collected serum every 6 months from individuals in whom Forced Expiratory Volume in 1 second (FEV1) \% predicted was $>80 \%$. We sought to correlate serial DES/IDES with CT density decline as measured by PD15, FEV1 decline, and determine the intra-subject test to test variability of DES/IDES.

\section{Methods}

\section{Study design and population}

QUANTUM-1 (NCT00532805) was funded to enroll 50 individuals. 49 participants were enrolled between 09/19/2007 and 12/11/2008 after an informed consent was obtained at one of the 7 U.S. participating study centers ( 1 individual was a screen-fail). Institutional board review at all enrolling institutions approved the study. All participants were protease inhibitor ZZ (PiZZ) with normal lung function at baseline visit as defined by post-bronchodilator (post-BD) FEV1 \% predicted $\geq 80 \%$ by National Health and Nutrition Examination Survey III (NHANES III) [18]. None were smokers during the study. All participants had telephone calls every two months to report exacerbations and serial site visits were completed (at baseline, 6, 12, 18, 24, and 36 months) with chest $\mathrm{CT}$, pulmonary function tests (spirometry, lung volumes, and diffusing capacity of the lung for carbon monoxide (DLCO)), and serum collection to explore the biomarker levels and their variability in the three year interval.

The exacerbation frequency for the year prior to the study was determined at the baseline visit using the AlphaNet exacerbation questionnaire $[19,20]$. This questionnaire was specifically developed to define exacerbation type and frequency by a variety of definitions and has been used in large cohorts of AATD individuals who have been receiving augmentation therapy through AlphaNet.

\section{CT acquisition and analysis}

In accord with the study protocol, $18 \mathrm{mcg}$ of tiotropium was administered with $180 \mu \mathrm{g}$ of albuterol for maximal bronchodilation at the time when the CT scan was performed (within 4 hours). Contiguous non-overlapping $0.6-1.0 \mathrm{~mm}$ slice thickness multidetector row CT scans were acquired at $80 \mathrm{mAs}, 120 \mathrm{kVp}$. These images were reconstructed using a medium spatial frequency reconstruction algorithm (GE: Standard, Philips: B, Siemens: B45f, Toshiba: FC86). All acceptable baseline and 36-month CT scans that could be analyzed using Apollo Image Analysis software (VIDA (Volumetric Imaging Display and Analysis) Diagnostics Inc.) were included in the analysis. Briefly, the lungs were segmented in three-dimensions from the chest wall and mediastinal components using Apollo. The CT-derived total lung volume (TLC), mean lung density and the lowest $15^{\text {th }}$ percentile density (PD15) corrected for lung volume were measured reflecting current practices [21]. Of the original 49 participants, 7 had missing data, and 11 had CT scans that did not follow the specific acquisition protocol. Thirty one (31) individuals remained eligible for analyses to correlate DES/IDES and emphysema progression. Baseline emphysema and DES/IDES levels were analyzed in 47 participants. Baseline PD15, PD15 slope, and the values adjusted for TLC were assessed as continuous variables.

\section{Pulmonary function tests}

Spirometry was performed before and after (20 minutes) administration of albuterol and tiotropium. Spirometers were calibrated to a volume standard on the day of testing. All expiratory efforts collected for the study were of minimum 6 second duration. The FEV1 and forced vital capacity (FVC) used for data collection were from the best efforts that were reproducible by 2 separate tests with $<5 \%$ and $<150 \mathrm{ml}$ variability. All subjects were retested serially on the same machines at each medical center to minimize test to test variability. Height was measured with shoes off at baseline visit.

\section{Blood samples}

$50 \mathrm{ml}$ of blood was drawn at initial study visit for serum, and plasma analysis. Serum samples were allowed to clot for 15 minutes per protocol, spun at $3500 \mathrm{rpm}$, and placed into a $-80^{\circ} \mathrm{C}$ freezer until transported on dry ice to the central repository.

\section{DES/IDES analysis}

The study individuals had longitudinal sampling of the blood for DES/IDES levels at all study visits. An average DES/IDES value was calculated for each study individual. Combined analyses of DES/IDES levels were performed in triplicates. The coefficient of variation of the method is $7 \%$ [22].

\section{Statistical analysis}

Continuous variables were evaluated by t-test and categorical variables were evaluated by chi-square test. A $p$-value $<0.05$ 
was considered significant. We analyzed correlations between demographics, FEV1 decline $(\mathrm{n}=49)$, baseline PD15/TLC $(\mathrm{n}=47)$ and PD15 slope $(n=31)$ for those with a complete dataset. A paired t-test was used to compare the values of DES/IDES at the beginning and at the end of the study in all of the participants. Least squares regression of FEV1 values defined FEV1 slope over 3-year period.

\section{Results}

\section{Patient characteristics}

The 49 participants had a follow-up $>90 \%$ for 6 study visits. The mean age was $51 \pm 10$, most were females (65\%). GOLD stage 1 obstruction was present in 10/49 individuals and FEV1/FVC post-BD was normal in 39/49 (mean FEV1/FVC 0.76). The mean FEV1 postbronchodilator was $99 \pm 11 \%$ predicted and subsequent 3-year FEV1 decline averaged $49 \mathrm{ml} /$ year.

CT of the chest was obtained in 31 study participants and radiographic data was used for statistical analysis. The baseline demographic and clinical characteristics of these resultant 2 group participants are summarized in table 1 . The two groups did not have any differences despite the fact that this separation was not prespecified in the original study protocol.

\section{Longitudinal changes of DES/IDES levels}

At the baseline visit, the mean and median levels of DES/IDES were $0.31 \pm 0.07 \mathrm{ng} / \mathrm{ml}$ and $0.30 \mathrm{ng} / \mathrm{ml}$ (range $0.20-0.48 \mathrm{ng} / \mathrm{ml}$ ) which are elevated over predicted normal mean levels of $0.19 \pm 0.01 \mathrm{ng} / \mathrm{ml}$. The average mean and median levels of DES/IDES were $0.30 \pm 0.07 \mathrm{ng} /$ $\mathrm{ml}$ and $0.29 \mathrm{ng} / \mathrm{ml}$ (range $0.20-0.49 \mathrm{ng} / \mathrm{ml}$ ) for all visits. This value is higher than the normal population $(0.19 \pm 0.1 \mathrm{ng} / \mathrm{ml})$ [17]. No gender differences were detected $(p=0.25)$ (Table 2$)$.

Baseline and average DES/IDES were highly correlated with patient age $\left(r^{2}=0.30\right.$ and 0.44 respectively, $p<0.0001$ for both). Over the 3 -year period, intra-individual DES/IDES variability was small. A matched pair's analysis at the patient level compared all potential pairs of serum values and found no statistical differences at any time (all $p>0.05$ ).

\section{Correlation of DES/IDES with FEV1 slope and DLCO}

Baseline and average DES/IDES did not correlate with FEV1 slope ( $r^{2}=0.0076$ and 0.0099 respectively, $p>0.49$ for both). Baseline and average DES/IDES correlated with baseline DLCO adjusted for hemoglobin ( $\mathrm{r}^{2}=0.10$ and 0.17 respectively, $p<0.02$ for both). Baseline and average DES/IDES did not correlate with DLCO at 36 months (N $=46)$ adjusted for hemoglobin $\left(\mathrm{r}^{2}=0.01\right.$ and 0.06 respectively, $p=0.40$ and $p=0.09$ ).

\section{Correlation of DES/IDES with emphysema and its progression}

Despite normal FEV1 \% predicted, emphysema was usually present on visual analysis. Mean baseline PD15 in 47 participants was $-937 \pm$ 25 Hounsfield Units (HU). Baseline serum DES/IDES did not correlate with baseline PD15 $\left(\mathrm{r}^{2}=0.02, \mathrm{p}=0.33\right)$. The mean DES/IDES over 3 years trended in the same direction as PD15 slope $\left(r^{2}=0.09, \mathrm{p}=0.09\right)$ in 31 individuals with a complete dataset. Baseline serum DES/IDES did not correlate with baseline PD15 adjusted for TLC $\left(\mathrm{r}^{2}=0.0002, \mathrm{p}\right.$ $=0.92$ ). The mean DES/IDES over 3 years did not correlate with PD15 slope adjusted for TLC $\left(\mathrm{r}^{2}=0.01, \mathrm{p}=0.57\right)$.

\section{Exacerbations}

The exacerbation questionnaire administered during the first study visit revealed that $47 \%$ of individuals had at least one exacerbation in the year preceding the study. This data was similar to findings in the SubPopulations and InteRmediate Outcome Measures In COPD Study (SPIROMICS) cohort in which individuals had exacerbations while not meeting current criteria for COPD diagnosis [23]. Most (69\%) had at least one exacerbation during the study period (7 individuals had missing data and were not included in the analysis). A subset of participants (36\%) who had two or more exacerbations per year were evaluated for DES/IDES changes. No differences in DES/IDES levels were detected between the group who had frequent exacerbations and the group with the annual rate of exacerbation less than two.

\section{Discussion}

This study demonstrates statistically significant increases in serum DES/IDES levels and stability of serial measurements of DES/IDES in AATD individuals with normal spirometry. DES/IDES are the unique products of elastin degradation, a determining component of emphysema development. Therefore, DES/IDES elevations are anticipated in emphysema progression.

COPD and AATD are characterized by persistent progressive limitation of airflow and enhanced lung inflammatory response to variety of noxious stimuli. However, elastin is also abundant in non-

Table 1. Baseline demographic and clinical features of the PiZZ alpha-1 antitrypsin deficiency cohort

\begin{tabular}{|c|c|c|c|c|c|}
\hline Characteristic & $\begin{array}{l}\text { FEV1 Slope Included Patients } \\
\qquad N=49\end{array}$ & Range & $\begin{array}{l}\text { PD15 Slope Included Patients } \\
\qquad(\mathrm{N}=31)\end{array}$ & Range & $P$ Value \\
\hline Age, years $\dagger$ & $51 \pm 10$ & $32-71$ & $52 \pm 10$ & $32-71$ & 0.45 \\
\hline Sex, number of females (\%) & $32(65 \%)$ & N/A & $18(58 \%)$ & N/A & 0.15 \\
\hline BMI, $\mathrm{kg} / \mathrm{m}^{2} \dagger$ & $29 \pm 7$ & $21-48$ & $30 \pm 7$ & $21-48$ & 0.24 \\
\hline FEV1 post-BD $\%$ predicted $\dagger$ & $99 \pm 11$ & $75-123$ & $101 \pm 12$ & $76-123$ & 0.22 \\
\hline FEV1/FVC post-BD & $0.76 \pm 0.1$ & $0.5-0.96$ & $0.76 \pm 0.1$ & $0.51-0.89$ & 0.95 \\
\hline Baseline $\mathrm{PD} 15, \mathrm{HU} \dagger(\mathrm{N}=47)$ & $-937 \pm 25$ & $-980 ;-846$ & $-93 \pm 27$ & $-980 ;-846$ & 0.99 \\
\hline Baseline PD15/TLC $(\mathrm{N}=47)$ & $-0.17 \pm 0.04$ & $-0.30 ;-0.1$ & $-0.17 \pm 0.04$ & $-0.26 ;-0.1$ & 0.31 \\
\hline Baseline DES/IDES, ng/ml & $0.31 \pm 0.07$ & $0.2 ; 0.48$ & $0.32 \pm 0.07$ & $0.2 ; 0.48$ & 0.45 \\
\hline
\end{tabular}

$\dagger$ tmean \pm Standard Deviation. Definition of abbreviations: BMI-body mass index; FEV1-forced expiratory volume in 1 second; post-BD-post bronchodilator; FVC-forced vital capacity; PD15-percentile density at 15\%; TLC-total lung capacity; DES-desmosine; IDES-isodesmosine

Table 2. Longitudinal changes of the PiZZ alpha-1 antitrypsin deficiency cohort. †mean \pm Standard Deviation

\begin{tabular}{|l|l|l|l|l|}
\hline Characteristic & $\begin{array}{l}\text { FEV1 Slope Included Patients } \\
\mathbf{N = 4 9}\end{array}$ & Range & $\begin{array}{l}\text { PD15 Slope Included Patients } \\
\text { (N=31) }\end{array}$ & Range \\
\hline Mean loss of FEV1, ml/year $\dagger$ & $49 \pm 86$ & $-300 ;+165$ & $58 \pm 85$ & $-298 ;+80$ \\
\hline Mean DES/IDES, ng/ml & $0.30 \pm 0.07$ & $0.2 ; 0.49$ & $0.31 \pm 0.07$ & 0.31 \\
\hline
\end{tabular}


pulmonary tissues and may result in cardiovascular disease (CVD), a common comorbidity of COPD [24]. CVD frequently complicates COPD and affects patient related outcomes, associated morbidity, and mortality. The ECLIPSE evaluation concluded that excess elastin degradation is associated with cardiovascular risk and mortality in COPD [25]. Similarly, about $30 \%$ of individuals with established CVD manifest airflow limitation and most likely have underdiagnosed COPD [26].

This study confirms the significant association of age and increased production of DES/IDES. Age is associated with an amplified degradation of body elastin although the mechanisms remain unclear. Recent studies of usual COPD explored these associations in two independent cohorts with much larger samples and concur with our findings [25]. We also noted that the serum concentrations in our cohort are higher than in other normal populations. The mean DES/ IDES averaged per patient over 3 years was higher than the normal population, possibly reflecting that AATD is a disease in which elastin degradation of all tissues can be accelerated. This significant elevation of DES/IDES in AATD population with nearly normal spirometry is an important finding further stressing the ongoing disease process despite normal FEV1.

Although we did not find a correlation between products of elastin degradation and radiographic emphysema progression, our sample was small, with young, non-smoking participants, and emphysema progression was very limited during the 3 years of observation. Another limitation of our study is the sample selection bias since there was no matched group of subjects with a PiMM genotype possessing the same clinical and demographic characteristics. A larger study of AATD individuals with more advanced airflow obstruction and radiographic emphysema receiving or not receiving augmentation therapy [14] evaluated the associations of DES/IDES and emphysema progression. For the first time it was shown that augmentation therapy, even if delayed, was associated with a decrease in elastin degradation and slower lung density decline [15]. DES/IDES levels were found to be significantly reduced after initiation of augmentation therapy at all of the points of the study (up to 4 years). The placebo group was found to have increased levels of DES/IDES from baseline at 2 years. This data further supports the use of DES/IDES levels as a biomarker to monitor the progression of emphysema and evaluate the treatment response. A study utilizing treatment with tiotropium aerosol also confirmed the role of DES/IDES as an end point of therapy [27].

Little is known about the natural history of exacerbations in AATD despite the central role they play in usual COPD outcomes. Current studies cannot show any impact on cost, trajectory of lung function, and other important patient related outcomes. Similarly, this study did not detect DES/IDES differences between individuals with frequent exacerbations and those without.

It is noteworthy that DES/IDES plasma levels were statistically correlated with severity of emphysema by CT in the Randomized placebo-controlled trial of Augmentation therapy in alpha-1 Proteinase Inhibitor Deficiency study (RAPID) in AATD, where emphysema is a predominant lesion. This is in contrast to usual COPD where the presence and severity of emphysema is highly variable [24]. Further studies on COPD phenotypes should be evaluating DES/IDES as a biomarker in emphysema-predominant COPD.

\section{Conclusions}

Mean DES/IDES levels in serum were higher in PiZZ individuals with FEV $1 \geq 80 \%$ predicted compared to historical cohorts of normal individuals. However, DES/IDES failed to correlate with baseline
PD15, PD15 decline, or FEV1 slope over 3 years in this study. The consistency of the $6 \mathrm{DES} / \mathrm{IDES}$ measurements in each patient adds knowledge about test to test variability in early AATD. Additionally, low variability between individual participants was noted. In summary, the longitudinal performance of DES/IDES is an important finding of our study and adds to its validation. The imbalance imposed between the continued activity of neutrophil elastase and the deficient levels of alpha-1 antitrypsin inhibition could lead to increased levels of elastin degradation in the lung and other organs at later stages of AADT. Recognizing increased elastin degradation at the earliest stages of AATD therapies may be warranted at the earliest in the clinical course. We hope that our findings may help formulate new hypotheses and lead to studies with greater statistical power.

\section{Summary}

Desmosine (DES) and Isodesmosine (IDES) are products of elastin degradation that increase in emphysema individuals. Previous studies confirmed the significant correlation of DES/IDES with lung function in moderate COPD. We demonstrate that despite normal spirometry, individuals with severe deficiency of alpha-1 antitrypsin have higher than normal levels of these biomarkers.

\section{Acknowledgements}

Author contributions: T.B. and C.S. had access to all study data and take responsibility for the integrity of the data and the accuracy of the analysis. T.B., S.M., C.S., and G.M.T. contributed to the study design, data analysis, and manuscript writing.

Other contributions: The QUANTUM-1 investigators responsible for participant enrollment included Alan Barker, Mark Brantly, Robert Sandhaus, Edwin Silverman, James Stoller, and Bruce Trapnell.

Funding support: QUANTUM-1 Study was supported by NHLBI, the Office of Rare Diseases through the Rare Lung Disease Clinical Research Network (1 U54 RR019498-01, Trapnell PI), and the Alpha-1 Foundation.

\section{References}

1. Zacherle E, Noone JM, Runken MC, Blanchette CM (2015) Health care cost and utilization associated with alpha- 1 antitrypsin deficiency among a cohort of medicare beneficiaries with COPD. Value Health 18: A664. [Crossref]

2. Stone PJ, Bryan-Rhadfi J, Lucey EC, Ciccolella DE, Crombie G, et al. (1991) Measurement of urinary desmosine by isotope dilution and high-performance liquid chromatography. Correlation between elastase-induced air-space enlargement in the hamster and elevation of urinary desmosine. Am Rev Respir Dis 144: 284-290. [Crossref]

3. Janoff A, Chanana AD, Joel DD, Susskind H, Laurent P, et al. (1983) Evaluation of the urinary desmosine radioimmunoassay as a monitor of lung injury after endobronchial elastase instillation in sheep. Am Rev Respir Dis 128: 545-551. [Crossref]

4. Stone PJ, Gottlieb DJ, O'Connor GT, Ciccolella DE, Breuer R, et al. (1995) Elastin and collagen degradation products in urine of smokers with and without chronic obstructive pulmonary disease. Am J Respir Crit Care Med 151: 952-959. [Crossref]

5. Gottlieb DJ, Stone PJ, Sparrow D, Gale ME, Weiss ST, et al. (1996) Urinary desmosine excretion in smokers with and without rapid decline of lung function: the Normative Aging Study. Am J Respir Crit Care Med 154: 1290-1295. [Crossref]

6. Fregonese L, Ferrari F, Fumagalli M, Luisetti M, Stolk J, et al. (2011) Long-term variability of desmosine/isodesmosine as biomarker in alpha-1-antritrypsin deficiencyrelated COPD. COPD 8: 329-333. [Crossref]

7. Lindberg CA, Engström G, de Verdier MG, Nihlén U, Anderson M, et al. (2012) Total desmosines in plasma and urine correlate with lung function. Eur Respir J 39: 839-845. [Crossref]

8. Huang JT, Chaudhuri R, Albarbarawi O, Barton A, Grierson C, et al. (2012) Clinical validity of plasma and urinary desmosine as biomarkers for chronic obstructive pulmonary disease. Thorax 67: 502-508. [Crossref] 
9. Wewers MD, Casolaro MA, Sellers SE, Swayze SC, McPhaul KM, et al. (1987) Replacement therapy for alpha 1-antitrypsin deficiency associated with emphysema. $N$ Engl J Med 316: 1055-1062. [Crossref]

10. Stone PJ, Morris TA, 3rd, Franzblau C, Snider GL (1995) Preliminary evidence that augmentation therapy diminishes degradation of cross-linked elastin in alpha-1antitrypsin-deficient humans. Respiration 62: 76-79. [Crossref]

11. Gottlieb DJ, Luisetti M, Stone PJ, Allegra L, Cantey-Kiser JM, et al. (2000) Shortterm supplementation therapy does not affect elastin degradation in severe alpha(1)antitrypsin deficiency. The American-Italian AATD Study Group. Am J Respir Crit Care Med 162: 2069-2072. [Crossref]

12. Ma S, Lin YY, He J, Rouhani FN, Brantly M, et al. (2013) Alpha-1 antitrypsin augmentation therapy and biomarkers of elastin degradation. COPD 10: 473-481. [Crossref]

13. Stoller JK, Rouhani F, Brantly M, Shahin S, Dweik RA, et al. (2002) Biochemical efficacy and safety of a new pooled human plasma alpha(1)-antitrypsin, Respitin. Chest 122: 6674. [Crossref]

14. Chapman KR, Burdon JG, Piitulainen E, Sandhaus RA, Seersholm N, et al. (2015) Intravenous augmentation treatment and lung density in severe $\alpha 1$ antitrypsin deficiency (RAPID): a randomised, double-blind, placebo-controlled trial. Lancet 386: 360-368. [Crossref]

15. Ma S, Lin YY, Cantor JO, Chapman KR, Sandhaus RA, et al. (2017) The effect of alpha-1 proteinase inhibitor on biomarkers of elastin degradation in alpha-1 antitrypsin deficiency: An analysis of the RAPID/RAPID Extension trials. Chronic Obstr Pulm Dis 4: 34-44. [Crossref]

16. Ongay S, Sikma M, Horvatovich P, Hermans J, Miller BE, et al. (2016) Free Urinary Desmosine and Isodesmosine as COPD Biomarkers: The Relevance of Confounding Factors. Chronic Obstr Pulm Dis 3: 560-569. [Crossref]

17. Ma S, Lin YY, Turino GM (2007) Measurements of desmosine and isodesmosine by mass spectrometry in COPD. Chest 131: 1363-1371. [Crossref]
18. Hankinson JL, Odencrantz JR, Fedan KB (1999) Spirometric reference values from a sample of the general U.S. population. Am J Respir Crit Care Med 159: 179-187. [Crossref]

19. Hoth KF, Ford DW, Sandhaus RA, Strange C, Wamboldt FS, et al. (2012) Alcohol use predicts ER visits in individuals with alpha-1 antitrypsin deficiency (AATD) associated COPD. COPD 9: 417-425. [Crossref]

20. Campos MA, Alazemi S, Zhang G, Wanner A, Salathe M, et al. (2009) Exacerbations in subjects with alpha-1 antitrypsin deficiency receiving augmentation therapy. Respir Med 103: 1532-1539. [Crossref]

21. Shaker SB, Dirksen A, Laursen LC, Skovgaard LT, Holstein-Rathlou NH (2004) Volume adjustment of lung density by computed tomography scans in patients with emphysema. Acta Radiol 45: 417-423. [Crossref]

22. Ma S, Turino GM, Hayashi T, Yanuma H, Usuki T, et al. (2013) Stable deuterium internal standard for the isotope-dilution LC-MS/MS analysis of elastin degradation. Anal Biochem 440: 158-165. [Crossref]

23. Woodruff PG, Barr RG, Bleecker E, Christenson SA, Couper D, et al. (2016) Clinical Significance of Symptoms in Smokers with Preserved Pulmonary Function. $N$ Engl $J$ Med 374: 1811-1821.

24. Curkendall SM, DeLuise C, Jones JK, Lanes S, Stang MR, et al. (2006) Cardiovascular disease in patients with chronic obstructive pulmonary disease, Saskatchewan Canada cardiovascular disease in COPD patients. Ann Epidemiol 16: 63-70. [Crossref]

25. Rabinovich RA, Miller BE, Wrobel K, Ranjit K, Williams MC, et al. (2016) Circulating desmosine levels do not predict emphysema progression but are associated with cardiovascular risk and mortality in COPD. Eur Respir J 47: 1365-1373. [Crossref]

26. Franssen FM, Soriano JB, Roche N, Bloomfield PH, Brusselle G, et al. (2016) Lung Function Abnormalities in Smokers with Ischemic Heart Disease. Am J Respir Crit Care Med 194: 568-576. [Crossref]

27. Ma S, Lin YY, Tartell L, Turino GM (2009) The effect of tiotropium therapy on markers of elastin degradation in COPD. Respir Res 10: 12. [Crossref]

Copyright: (C2018 Beiko T. This is an open-access article distributed under the terms of the Creative Commons Attribution License, which permits unrestricted use, distribution, and reproduction in any medium, provided the original author and source are credited. 